ARTICLE

\title{
MSI2 is required for maintaining activated myelodysplastic syndrome stem cells
}

James Taggart ${ }^{1, \star}$, Tzu-Chieh Ho ${ }^{1, \star}$, Elianna Amin ${ }^{1, \star}$, Haiming $\mathrm{Xu}^{2, \star}$, Trevor S. Barlowe ${ }^{1}$, Alexendar R. Perez ${ }^{3}$,

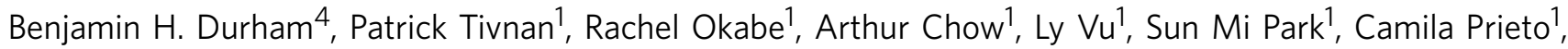
Christopher Famulare ${ }^{5}$, Minal Patel ${ }^{5}$, Christopher J. Lengner ${ }^{6}$, Amit Verma ${ }^{7}$, Gail Roboz ${ }^{8}$, Monica Guzman ${ }^{9}$, Virginia M. Klimek ${ }^{5}$, Omar Abdel-Wahab ${ }^{4}$, Christina Leslie ${ }^{3}$, Stephen D. Nimer ${ }^{10}$ \& Michael G. Kharas ${ }^{1}$

Myelodysplastic syndromes (MDS) are driven by complex genetic and epigenetic alterations. The MSI2 RNA-binding protein has been demonstrated to have a role in acute myeloid leukaemia and stem cell function, but its role in MDS is unknown. Here, we demonstrate that elevated MSI2 expression correlates with poor survival in MDS. Conditional deletion of Msi2 in a mouse model of MDS results in a rapid loss of MDS haematopoietic stem and progenitor cells (HSPCS) and reverses the clinical features of MDS. Inversely, inducible overexpression of MSI2 drives myeloid disease progression. The MDS HSPCs remain dependent on MSI2 expression after disease initiation. Furthermore, MSI2 expression expands and maintains a more activated (G1) MDS HSPC. Gene expression profiling of HSPCs from the MSI2 MDS mice identifies a signature that correlates with poor survival in MDS patients. Overall, we identify a role for MSI2 in MDS representing a therapeutic target in this disease.

\footnotetext{
${ }^{1}$ Molecular Pharmacology and Center for Cell Engineering, Center for Stem Cell Biology, Center for Experimental Therapeutics, Memorial Sloan Kettering Cancer Center, New York, New York 10065, USA. ${ }^{2}$ Memorial Sloan Kettering Cancer Center, Cancer Biology Program, New York, New York 10065, USA. ${ }^{3}$ Computational Biology Program Memorial Sloan Kettering Cancer Center, Memorial Sloan Kettering Institute, New York, New York 10065, USA. ${ }^{4}$ Memorial Sloan Kettering Cancer Center, Human Oncology and Pathogenesis Program, New York, New York 10065, USA. ${ }^{5}$ Memorial Sloan Kettering Cancer Center, Department of Medicine, Leukemia Service, New York, New York 10065, USA. ${ }^{6}$ Department of Animal Biology, Department of Cell and Developmental Biology and Institute for Regenerative Medicine, Schools of Veterinary Medicine and Medicine, University of Pennsylvania, Philadelphia, Pennsylvania 19104, USA. ${ }^{7}$ Montefiore Medical Center, Albert Einstein College of Medicine, Bronx, New York 10461, USA. ${ }^{8}$ Joan and Sanford I. Weill Department of Medicine, Weill Cornell Medical College, New York, New York 10065, USA. ${ }^{9}$ Division of Hematology and Medical Oncology, Department of Medicine and Pharmacology, Weill Cornell Medical College, Cornell University, New York, New York 10065, USA. ${ }^{10}$ Sylvester Comprehensive Cancer Center, Department of Medicine, Miller School of Medicine, University of Miami, Miami, Florida 33136, USA. ${ }^{\star}$ These authors contributed equally to this work. Correspondence and requests for materials should be addressed to M.G.K. (email: Kharasm@mskcc.org).
} 
T he majority of haematological disorders involving the myeloid lineage are thought to be of stem cell origin, including myeloproliferative diseases, myelodysplastic syndromes, acute myeloid leukaemia and acquired or heritable bone marrow failure syndromes ${ }^{1-3}$. In each instance, dysregulation of normal stem cell function is thought to contribute to the disease phenotype. Moreover, stem cell characteristics are modulated by a variety of developmental pathways and regulators. Recent studies of MSI2 in normal and malignant hematopoietic stem cell (HSC) biology suggested that MSI2 might play a role in myelodysplastic syndromes (MDS) ${ }^{4-11}$. It was previously reported that MSI2 expression in MDS was reduced in patients with low-risk and high-risk MDS compared with normal CD34 cells ${ }^{7}$. However, in this study there was a subset of MDS patients with excess blasts with increased MSI2 (ref. 7). The functional importance of MSI2 in MDS therefore remains unclear. We examine previously published expression data sets and patient samples to find that MSI2 is increased in high-risk MDS patients. Additionally, we utilize MSI2 loss and gain of function approaches in the context of a mouse model of MDS and find that MSI2 is required for MDS.

\section{Results}

Elevated MSI2 expression predicts poor survival in MDS. In our examination of a previously published expression data set, we found that MSI2 expression was increased in $\mathrm{CD} 34^{+}$population in high-risk MDS patients (refractroy anemia with excess blasts; RAEB) compared with healthy individuals that were not age matched or Low-Risk MDS (Refractory Anemia; RA or refractory anemia with ringed sideroblasts; RARS), Fig. 1a) ${ }^{12}$. Elevated MSI2 levels correlated with a poor clinical survival (Fig. 1b and Supplementary Fig. 1a). In line with the microarray data, highrisk MDS patients had increased intracellular MSI2 in their $\mathrm{CD} 34^{+} \mathrm{CD}_{38}{ }^{-}$cells compared with low-risk MDS patients and healthy individuals (Fig. 1c,d). Altogether, the MDS patient data suggests that the level of MSI2 expression correlates with disease subtype and clinical outcome. In contrast to the acute myelogenous leukemia (AML) patient data, where elevated MSI2 expression correlates with FLT3-ITD/NPM1 mutations $5,8,9,11$, MDS patients do not typically harbour these mutations. Due to the low number of patients with recurrent mutations in this study, we are unable to correlate MSI2 levels with individual mutations (Supplementary Table 1).

Msi2 is required for MDS. To test if Msi2 could be functionally important in MDS, we utilized a murine model of MDS. The NUP98-HOXD13 transgenic model (NHD13) recapitulates many of the salient features of MDS, including neutropenia, lymphopenia and hypercellular or normocellular bone marrow at 4-7 months ${ }^{13-16}$. Also, $12-17 \%$ of the marrow contains dysplastic erythroid, myeloid and rare megakaryocytic cell types ${ }^{13}$. Similar to patients with MDS, a significant cohort of the primary mice can progress and develop an aggressive AML. However, if the bone marrow of NHD13 mice is transplanted, the recipient animals succumb to a fully penetrant but non-lethal form of MDS that rarely progresses to AML (ref. 15). Although the NHD13 a

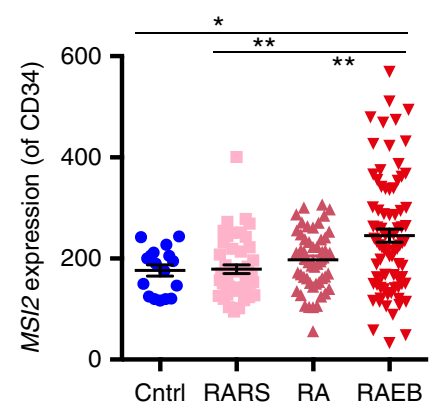

b

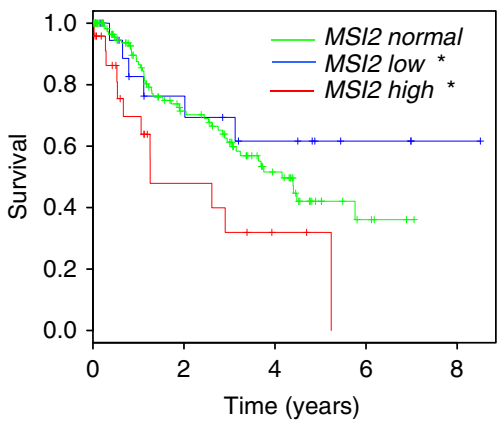

c

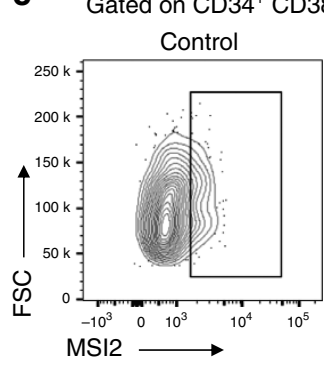

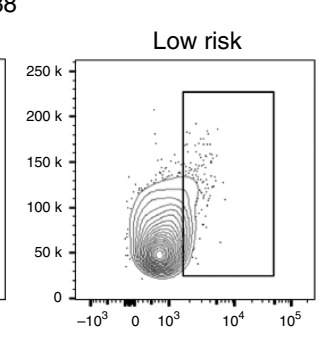

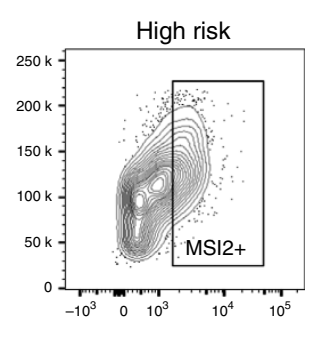

d

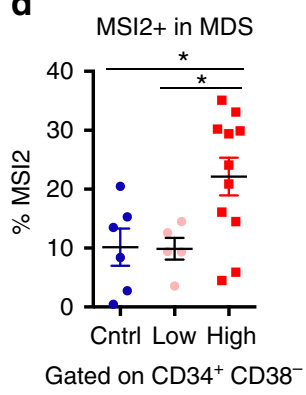

e

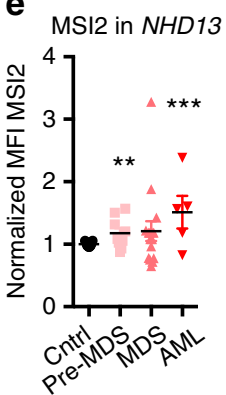

Figure 1 | Elevated MSI2 expression predicts poor survival in MDS. (a) Microarray expression data (CD34 ${ }^{+}$population) from normal elderly individuals $(C D 34+; n=17)$ and MDS $(n=183)$, RARS, RA, RAEB, ${ }^{\star} P<0.05,{ }^{\star \star} P<0.01,{ }^{\star \star \star} P<0.001$ Student's $t$-test mean \pm s.e. $\mathrm{m}^{12}$. (b) Overall survival in MDS patients stratified by MSI2 expression (as high ( $Z$-score $>1$ ), low ( $Z$-score $<-1$ ), or normal $(-1 \leq Z$-score $\leq 1)$ log-rank test. (c). Representative flow cytometric analysis of independent patient cohort of primary patients samples gated on CD34+CD38 ${ }^{-}$and stained for intracellular MSI2, age-matched elderly individuals $(n=6)$, low risk (RA or RARS; $n=5$ ) and high risk (RAEB-1, RAEB- $n=10)($ d). MSI2 positivity summarized from gating of patients in c. (e) MSI2 intracellular levels in the NHD13 MDS/AML animal model. Cells are initially gated on MSI2-positive cells (Supplementary Fig. 1 for gating) and median fluorescence intensity (MFI) is normalized to the control (C57BL6 mice), $n=19$. Pre-MDS represents the analysis preformed in NHD13 primary or transplanted mice within 1-2 months of birth and before MDS onset $(n=9)$, the MDS mice that are older than 2 months or primary transplanted and have low WBC, $(n=16)$ and AML samples are from NHD13 mice that have transformed to AML $(n=5), \mathbf{d}$ and $\mathbf{e}^{\star} P<0.05,{ }^{\star \star} P<0.01,{ }^{\star \star \star} P<0.001$ Student's $t$-test horizontal line is the mean \pm s.e.m. 
transplanted bone marrow cells engraft poorly, they still retain the clinical features of MDS $(\sim 10-20 \%$ peripheral blood chimerism $)^{15}$. Utilizing intracellular staining for MSI2, we found a significant albeit modest increase in MSI2 levels in the bone marrow of $44 \%$ of NHD13 pre-MDS, $50 \%$ of MDS, and $80 \%$ of AML animals (Fig. 1e and Supplementary Fig. 1b). The significant increase in MSI2 was also observed within the sorted progenitors from pre-MDS animals (Supplementary Fig. 1c,d).

In agreement with MDS patient data, we observed an increase in the expression of MSI2 in the NHD13 mice during disease progression. These data suggested that altering MSI2 levels in the NHD13 model could alter the disease fate. To test this hypothesis, Msi2 conditional knockout were crossed with the NHD13 mice and then transplanted into congenic recipients (Fig. 2a,b). The chimerism in the peripheral blood and at the level of the haematopoietic stem and progenitor cell (HSPC) was significantly reduced one month after pIpC-mediated deletion (Fig. 2c,d). Msi2 deletion resulted in the loss of the NHD13-expressing cells and a reversal of MDS-like disease that included an increase in white blood cell (WBC) counts, red blood cells and platelets (Fig. 2e-g). When the mice were analyzed 14 months after transplantation there was a trend towards reduced spleen weight, normalized WBC counts and significantly reduced chimerism in the HSPCs and in progenitors (Supplementary Fig. 2a-c). Despite the fact that some of the mice had detectable donor chimerism, the donor myeloid cells were mainly absent and the few remaining donor cells retained MSI2 expression indicating that the deleted cells were selected against (Supplementary Fig. 2d,e). Nevertheless, these mice did not have detectable dysplastic cells or leukaemia in their bone marrow (Supplementary Fig. 2f).

MSI2 overexpression in MDS drives transformation. We next assessed if forced MSI2 expression could alter the disease course using the same model of MDS. Of note, MSI2 overexpression by itself does not result in leukaemic transformation ${ }^{5}$. We utilized our previously described inducible MSI2 overexpressing mouse model that can be controlled with doxycycline (KH2-Col1A1-tet-on-MSI2/ROSA26-rTTA) and crossed them with the NHD13 mice ${ }^{5}$. Control (C57BL/6), NHD13 or NHD13/ MSI2 bone marrow was transplanted into congenic mice and then allowed to engraft before MSI2 was induced (Fig. 3a). After 5 months the NHD13/MSI2 overexpression mice started to succumb to lethal myeloid diseases while the NHD13 mice had symptoms of a mild MDS. The NHD13/MSI2 mice had reduced WBC $(5 / 25)$ or elevated WBC counts, reduced red blood cell counts, increased mean corpuscular volume and increased chimerism in the blood at 5 months post-transplantation compared with the control and the NHD13 mice (Fig. 3b-e). We observed increased immature myeloid cells in the peripheral blood, and all of the MSI2 overexpressing NHD13 mice eventually succumbed to various lethal myeloid diseases including MPN/MDS or an AML/MDS with a median latency of 228 days (Fig. 3f-h and Supplementary Fig. 3a-g). At end point, we found that the NHD13/MSI2 mice had a more severe disease burden based on increased spleen and liver weights compared with the control and NHD13 mice (Fig. 3i,j). The NHD13 mice a

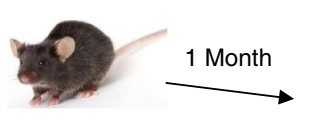

NHD13/Msi2 ${ }^{\mathrm{f} / \mathrm{f}}$

NHD13/Msi2 ${ }^{\mathrm{f} / \mathrm{f}} \mathrm{Mx1}$-Cre

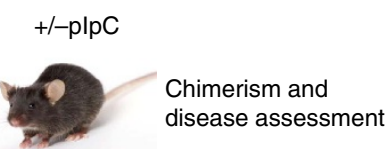

CD45.1 b

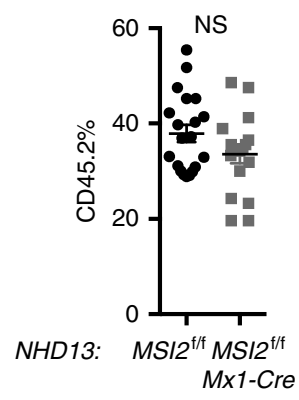

f

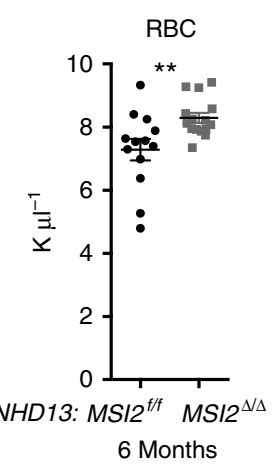

g

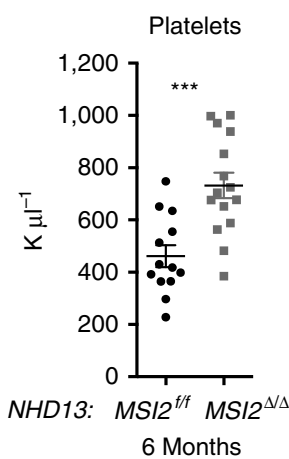

Figure 2 | Msi2 is required to maintain MDS. (a) Transplant scheme for Msi2 conditional knockout in NHD13 MDS model. (b) Peripheral blood chimerism 4 weeks posttransplantation as described in a, (NHD13/Msi2 ${ }^{f / f} n=20$ and NHD13/MSI2f/f Mx1-Cre; $\left.n=18\right)$. (c) Peripheral blood chimerism 8-weeks posttransplantation (4-weeks post-plpC treatment; NHD13/Msi ${ }^{f / f} n=15$ and NHD13/Msi2 $\left.{ }^{4 / \Delta} M x 1-C r e ; ~ n=14\right)$. (d) Chimerism within the haematopoietic stem and progenitor cell compartment from bone marrow aspirates performed 8-weeks posttransplantation, gated on Lin-Sca ${ }^{+} \mathrm{Kit}^{+} \mathrm{cells}_{\mathrm{s}}\left(\mathrm{NHD13} / \mathrm{Msi2}{ }^{\mathrm{f} / \mathrm{f}}\right.$ $n=12$ and $\left.N H D 13 / M s i 2^{4 / 4} M x 1-C r e ; n=10\right)$. (e) WBC. (f) Red blood cells (RBC). (g) Platelets at 6 months posttransplant, $\mathbf{f}-\mathbf{h}$, (analysed at 6 months postplpC, NHD13/Msi2 ${ }^{f / f} n=13$ and NHD13/Msi2 $\left.{ }^{4 / \Delta} M x 1-C r e ; n=15\right)$. Data in b-g are represented two independent transplants ${ }^{\star} P<0.05,{ }^{\star \star} P<0.01$,

${ }^{\star \star \star} P<0.001$, Student's $t$-test horizontal line is the mean \pm s.e.m. 
a

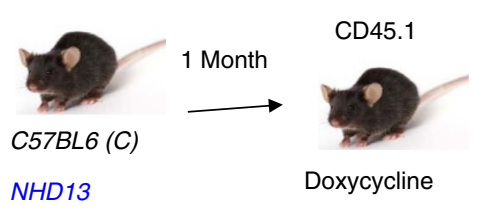

NHD13/MSI2 b

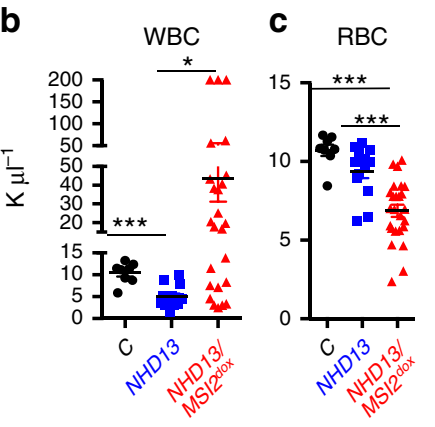

g

f

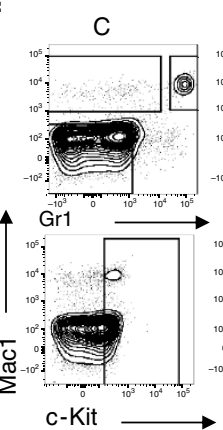

NHD13 NHD13/MSI2dox
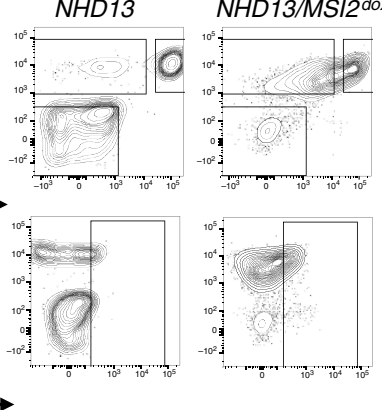

5 Month peripheral blood

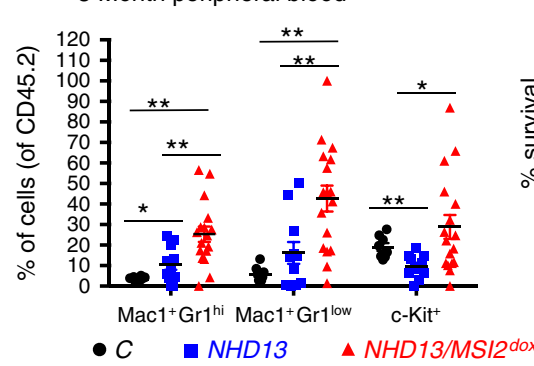

h
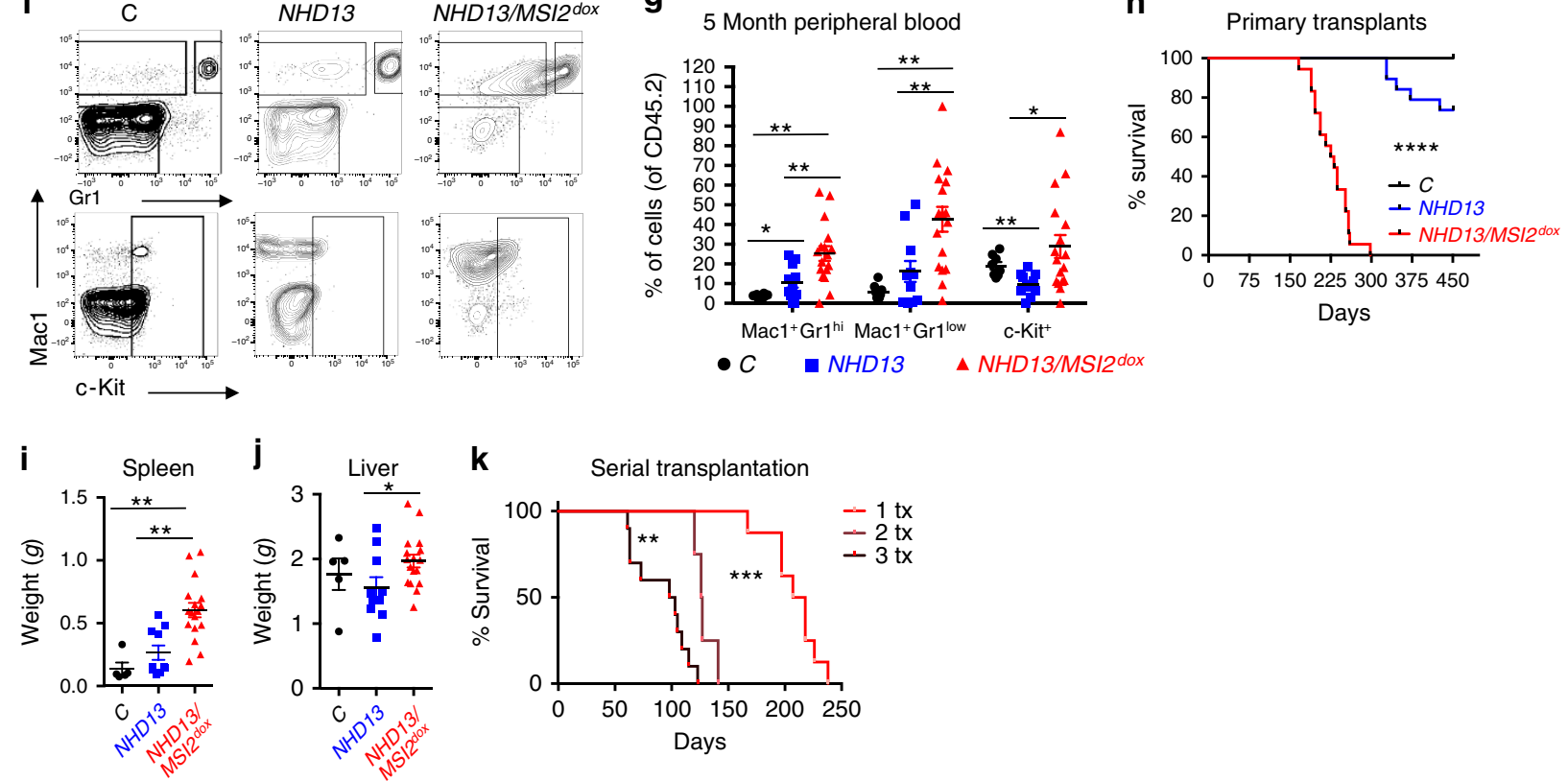

k

Serial transplantation

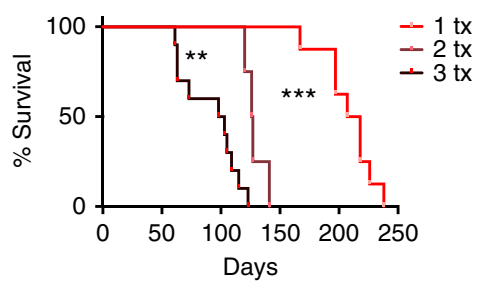

Figure 3 | Sustained MSI2 overexpression transforms MDS to a lethal AML. (a) Experimental scheme for MSI2 overexpression in the NHD13 transplant model. (b) Peripheral blood analysis 5 months posttransplantation, including WBC. (c) Red blood cell count (RBC). (d) Mean corpuscular volume (MCV). Data in b-d, (C; $n=8$, NHD13; $n=13, N H D 13 / M S I 2 ; n=25)$. (e) Chimerism (CD45.2) in the peripheral blood 5 months posttransplantation, (C; $n=8$, NHD13; $n=11, N H D 13 / M S 12 ; n=21$ ). (f) Representative Mac1, Gr1, and c-Kit staining of peripheral blood from the bone marrow. Mice were analysed 5 months posttransplant and gated on $\mathrm{CD} 45.2^{+}$cells. (g) Immunophenotyping of peripheral blood 5 months posttransplant. Samples gated as in $\mathbf{e}$, data in $\mathbf{f}$ and $\mathbf{g},(C ; n=8, N H D 13 ; n=11, N H D 13 / M S I 2 ; n=17)$. (h) Survival curves of NHD13 with MSI2 overexpression combined from two independent transplants mice ( $\left.; ; n=8, N H D 13 ; n=15, N H D 13 / M S 12^{D o x} ; n=18\right)$. (i) Spleen and (j) liver weights from healthy mice analysed at the end point or moribund mice, (C; $n=5, N H D 13 ; n=10, N H D 13 / M S 12 ; n=17$ ). (k) Survival analysis of serially transplanted myeloid disease in the NHD13/MSI2 Dox mice (primary donor; $n=8$, secondary; $n=4$ and tertiary transplants; $n=10$; transplanted from two independent donors). (I) Experimental scheme for testing MSI2 dependence of NHD13/MSI2 AML. The bone marrow from moribund primary NHD13/MSI2 transplanted animals was secondarily transplanted into congenic mice and treated accordingly. (m) Survival analysis for doxycycline on/off secondary transplants, (Dox ON; $n=8$ and Dox ${ }^{\text {OFF; }} n=8$ from two independent donors and transplants), Arrows 1, 2 and 3 indicate three representative mice that are described in $\mathbf{n}$ and $\mathbf{0}$. (n) Intracellular MSI2 staining by flow cytometry in fixed bone marrows of representative moribund mice described in $\mathbf{m}$. (o) Representative CD45.1/2 staining of mice at different points in the survival curve from $\mathbf{m}$. (p) Survival analysis of doxycycline treated NHD13/MSI2 secondary transplants relative to transplants whose recipients had a 2-week delay in doxycycline treatment posttransplant, Dox ${ }^{\mathrm{N}} ; n=9$ and Dox OFF; $n=10$ from two independent transplants). Data in b-k, are represented two independent transplants ${ }^{\star} P<0.05,{ }^{\star \star} P<0.01,{ }^{\star \star \star} P<0.001, \mathbf{b}-\mathbf{e}, \mathbf{g}, \mathbf{i}$ and $\mathbf{j}$ are Student's $t$-test and horizontal line is the mean \pm s.e.m and $\mathbf{h}, \mathbf{k}, \mathbf{m}$ and $\mathbf{p}$, $P$ values assessed by log-rank.

showed signs of a MPN/MDS or MDS disease, but only 3 out 15 mice died of a characterized myeloid disease (AML/MDS $n=2$, MDS $n=1$, and one mouse was found dead and another died of a non-myeloid disease; Fig. 3i,j). Serial transplantation of the NHD13/MSI2 demonstrated reduced latency further supporting the idea that MSI2 overexpression resulted in a clonal myeloid disease (Fig. 3k and Supplementary Fig. 3h). We secondarily transplanted the NHD13 AMLs and then compared the disease burden to the secondary transplants from the NHD13/MSI2 mice. Despite the fact that both groups had myeloid disease, the NHD13/MSI2 group retained their more aggressive phenotype compared with the NHD13 AMLs indicated by the increased spleen and liver weights (Supplementary Fig. 3i,j).

MSI2 maintains activated MDS stem and progenitor cells. We then determined if MSI2 overexpression was required to maintain the disease. Thus, we transplanted NHD13/MSI2 overexpressing mice into secondary recipients with or without doxycycline feed. Mice that were maintained on doxycycline and expressed MSI2 rapidly formed a lethal myeloid disease, while the majority of mice that were no longer being induced survived significantly 

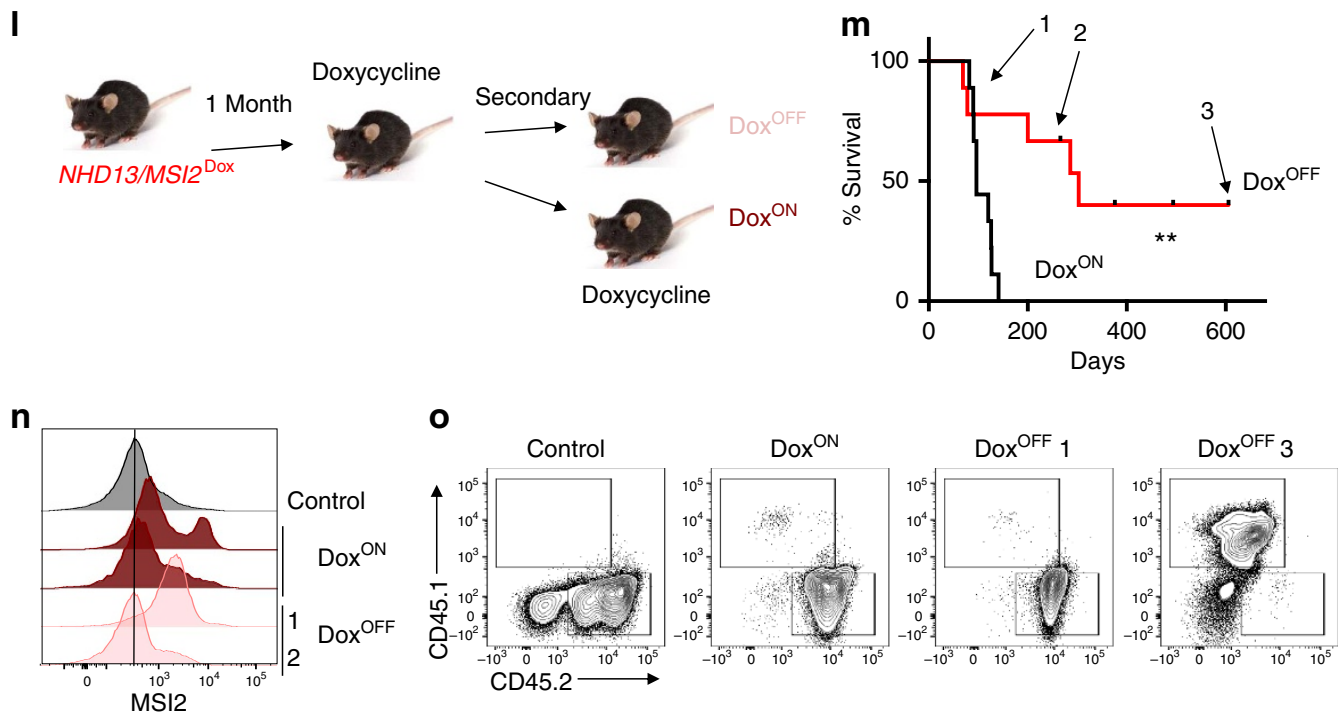

$\mathbf{0}$
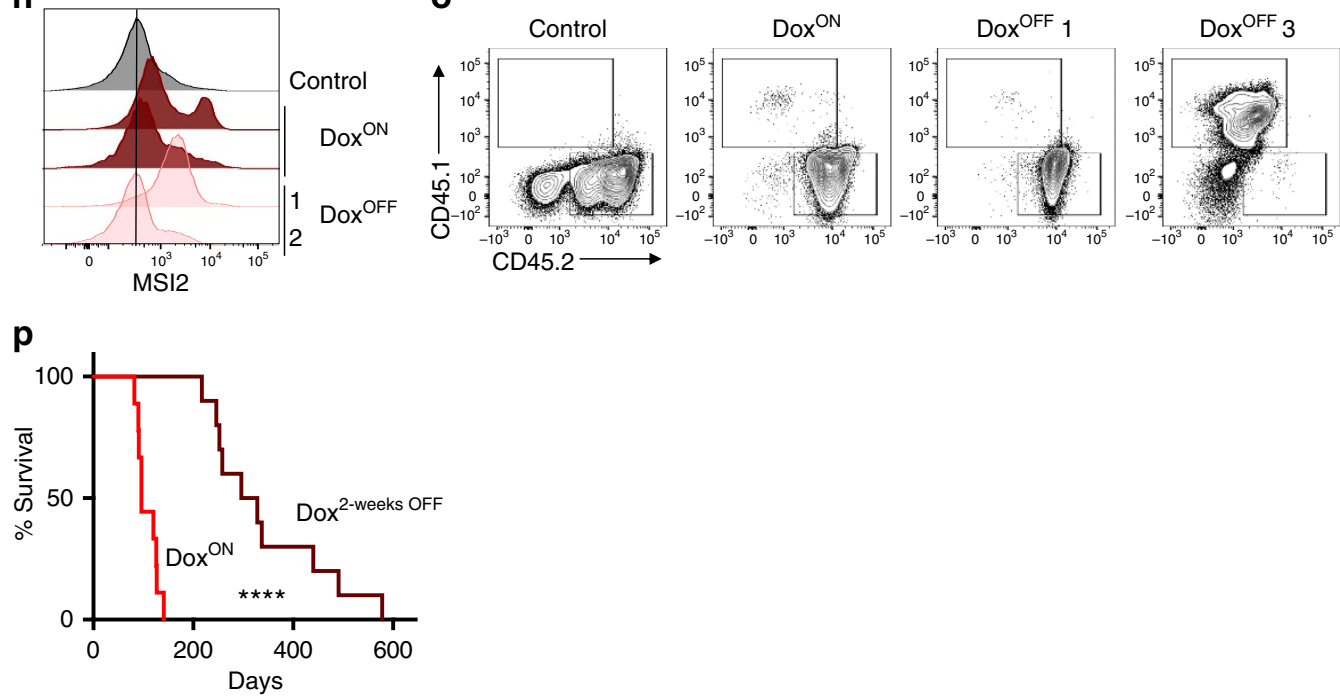

Figure 3 | continued.

longer (median, 96 versus 303 days; Fig. 31,m and Supplementary Fig. $3 k-t)$. Moreover, in mice that were no longer induced and died at the same time as the mice in the induced group, we were still able to detect high levels of intracellular MSI2 suggesting selection for constitutive activation in vivo (Fig. 3n). Similarly, a mouse that died later also demonstrated leaky MSI2 expression albeit at lower levels compared with the mouse that died earlier. Overall, in all the mice that died of leukaemia, MSI2-positive cells were detectable. However, mice killed at the experimental end point that remained disease free, we found that the chimerism was either low or undetectable (Fig. 3o). To further examine if transient withdrawal of MSI2 expression could also delay the leukaemia, we transplanted NHD13/MSI2 cells and waited 2 weeks to induce MSI2 expression (Fig. 3p). We observed a delay in the myeloid leukaemia (312 days compared with 96 days) in the control, and these leukaemias relapsed with MSI2 positivity, providing evidence that MSI2 overexpression must be sustained to maintain disease.

NHD13 haematopoietic cells have a block in their differentiation at the HSC to multipotent progenitors (MPP) stage and have dramatically reduced numbers of HSPCs (ref. 16; Fig. 4a,b). MSI2 induction for 5 days resulted in an increase in the percentage of phenotypic LSKs (Lin-Scal ${ }^{+} \mathrm{Kit}^{+}$cells) and a decrease in the phenotypic HSCs, but no difference in the frequency of the myeloid or erythroid progenitors (Fig. 4a-c and Supplementary Fig. 4a). Interestingly, if the 5-day-induced NHD13/MSI2 cells were then transplanted in the absence of doxycycline to turn off MSI2, we observed reduced chimerism at 1 month (Fig. 4d). Alternatively, when non-induced bone marrow was engrafted and then activated for MSI2, the LSK compartment was expanded at 1 month (Supplementary Fig. 4b), and in the diseased NHD13/ MSI2 mice LSKs and myeloid progenitors (granulocyte-monocyte progenitor (GMP) and common myeloid progenitor (CMP)) were increased compared with the NHD13 mice (Fig. 4e and Supplementary Fig. 4c). Similarly to the 5-day induction the chimerism of the phenotypic $\mathrm{HSCs}\left(\mathrm{LSK}^{+} \mathrm{CD} 150^{+}\right.$;CD48) was reduced in the diseased NHD13/MSI2 compared with the NHD13 and the control animals. We then profiled the cell cycle status of the HSPCs using Brdu incorporation and Hoechst staining and found reduced cell death (sub-G1) and increased percentage of cells in G1, which suggests the accumulation of more activated HSPCs (Fig. 4f,g). Taken together with the previous data, MSI2 expression maintains a more aggressive myeloid disease and a more activated HSPC.

To further characterize how MSI2 alters the NHD13 MDS programme in the dysregulated stem cell compartment, we performed transcriptome profiling in the HSPCs (LSK) from transplanted mice after 3 months of doxycycline administration and before the mice demonstrate any disease phenotype. To elucidate the NHD13/MSI2 expression programme, we utilized a generalized linear model that identified 891 significant genes (q-value $<0.01$, generalized linear model), of which 137 genes were upregulated ( $\log 2$ fold change $>0$ ) and 754 genes were downregulated ( $\log 2$ fold change $\leq 0)$. We then matched the gene signature to human homologues (690 genes; Supplementary Data 1) and created a heatmap after unsupervised hierarchical clustering, which separated the samples into their respective groups (Fig. 4h).

To functionally annotate our RNA-sequencing, we performed gene set enrichment analysis ${ }^{17}$ on all curated gene sets in the molecular signatures database (http://www.broadinstitute.org/ msigdb; 3,256 gene sets) combined with an additional set of relevant gene sets ( 92 gene sets from our experimentally derived or published haematopoietic self-renewal and differentiation signatures ${ }^{4,17}$; rank list; Supplementary Data 2). We found 14 gene sets that were enriched for genes that were upregulated and 
a

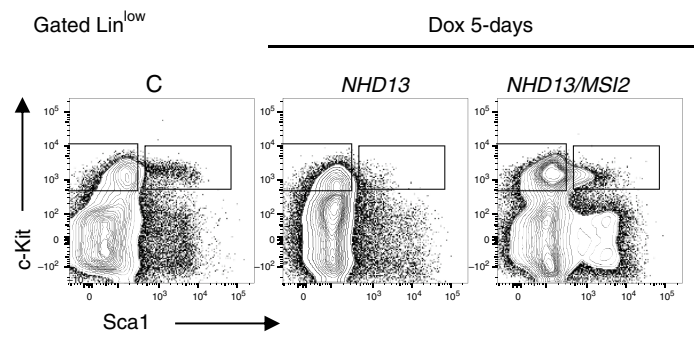

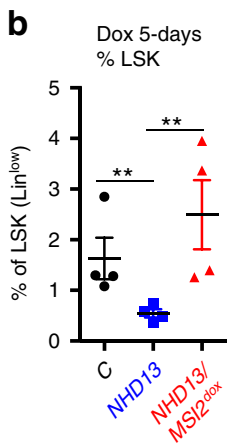

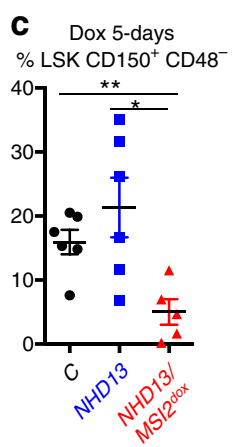

d

LSK chimerism 1 month

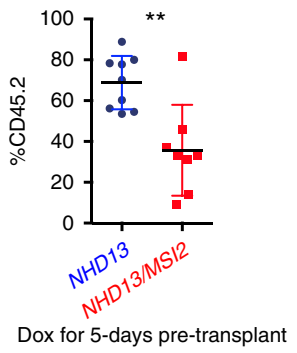

e

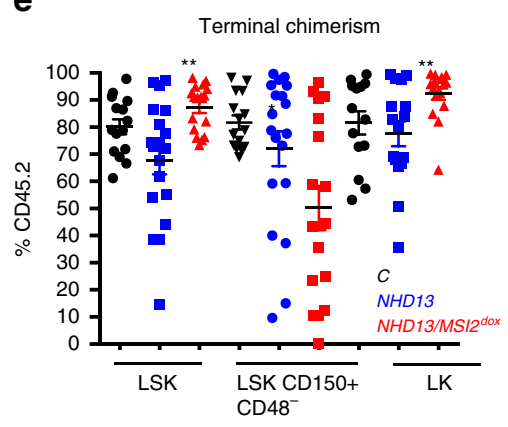

$\mathbf{f}$

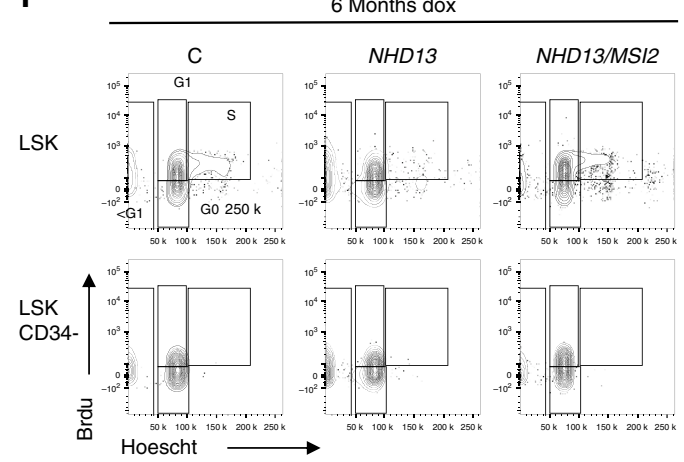

g

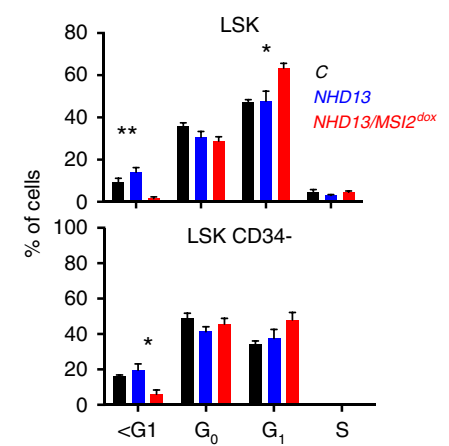

Figure 4 | MSI2 overexpression leads to haematopoietic progenitor stem cell expansion. (a) Representative flow cytometry of primary animals treated with doxycycline (Dox) for 5 days and live and Lin ${ }^{-}$gated. (b) Percentage of LSK population among the Lin ${ }^{\text {low }}$ compartment from primary mice (C; NHD13 and NHD13/MSI2 ${ }^{\text {Dox }} ; n=6, n=6$ and $n=5$ from five independent experiments). (c) Percentage of the HSC (LSK ${ }^{+} \mathrm{CD}_{150}{ }^{+} \mathrm{CD} 48^{-}$) compartment gated from the $\mathrm{LSK}^{+}$at 1 month transplantation after 5 day dox administration in the primary animals, data representative (C; NHD13 and NHD13/MSI2 ${ }^{\text {Dox }}$ same as b). (d) Chimerism (CD45.2) in the LSK compartment at 1 month transplantation after 5 day dox administration in the primary animals, data representative of two independent transplants (NHD13; $n=9$, NHD13/MSI2Dox; $n=8)$, (e) Terminal chimerism from transplants in Fig. 3 in the gated populations, (C,NHD13, NHD13/MSI2 Dox; $n=11-16$ combined from two independent transplants). (f) Representative flow cytometric plots from transplanted mice analysed (combined experiments from 3 and 7 months posttransplant) that were injected with Brdu $24 \mathrm{~h}$ and then sorted for LSK cells and gated accordingly. (g) Data represented in $\mathbf{f},(n=3$ for each group combined from two independent experiments). (h) RNA-sequencing of LSK sorted cells from primary transplanted animals ( 4 months posttransplantation and 3 months post MSI2 induction) before disease initiation underwent unsupervised clustering of the differentially expressed genes with human homologues, $\left(C ; n=3, N H D 13 ; n=2, N H D 13 / M S I 2^{\text {Dox }} ; n=4\right)$. (i-k) Gene set enrichment analysis (GSEA) from ranked list of NHD13/MSI2 Dox /NHD13 versus Control. (I) Unsupervised clustering of the mouse NHD13 signature overlapped with MDS patients. (m) MSI2 expression (Z-score) separated based on clustering in $\mathbf{I}$. (n) Survival of MDS patients based on clusters from the NHD13/MSI2 signature $(\mathbf{I}, \mathbf{m})$. Data in $\mathbf{b}-\mathbf{e}, \mathbf{g}$ and $\mathbf{m}{ }^{\star} P<0.05,{ }^{\star \star} P<0.01,{ }^{\star \star \star} P<0.001$, are Student's $t$-test and the horizontal line is the mean \pm s.e.m and $\mathbf{n}$, $P$ values are displayed and calculated with log-rank.

29 gene sets enriched for downregulated genes (Supplementary Tables 2 and 3). The top ranked gene sets included enrichment in an NRAS activated signature ${ }^{18}$, a reduced quiescent phenotype $\mathrm{e}^{19}$ and a more progenitor-like cell (Fig. $4 \mathrm{i}-\mathrm{k}$ ). Taken together with our phenotypic analysis of the HSPC compartment, MSI2 induction increases the cells that are in G1, switching them to a less quiescent and more progenitor-like gene expression signature.

To determine if the MSI2 signature from the murine model of MDS corresponds to patients with MDS, we overlapped the NHD13/MSI2 RNA-seq (690 genes) with microarray data from control $(n=17)$ and MDS patients $(n=183)$ (refs 12,20). After unsupervised clustering of the human microarray data, we obtained four distinct clusters (Fig. 4l). Patients with elevated MSI2 expression were mainly found in Cluster-2, which predicted a poor survival compared with the other clusters (Fig. $4 \mathrm{~m}, \mathrm{n}$ ). Our study demonstrates an important functional role of MSI2 in MDS (Supplementary Fig. 4d).

\section{Discussion}

In summary, we found that elevated MSI2 expression predicts poor prognosis in MDS and is required for maintaining the diseased MDS stem cell. Cooperativity with NHD13 has been associated with various factors including FLT3, MEIS1, P16 and TP53 (refs 16,21-23). MSI2 overexpression can act as a cooperating oncogene and drive transformation, accelerate leukaemia and increase disease burden in the context of a MDS mouse model. Additionally, we found reduced apoptosis and a more activated stem cell, suggesting that the altered HSPC may contribute to disease progression. Gene expression profiling of HSPCs from the NHD13/MSI2 mice generated a signature that overlapped with human MDS and could predict patient outcome. Our lab previously found that MSI2 directly binds to the mRNA of mixed-lineage leukaemia target genes including Hoxa9, Myc and $I k z f 2$, and regulates the translation of these targets in a mixed-lineage leukaemia-AF9 leukaemia model ${ }^{9}$. Additionally, a recent report showed that Msi2 may regulate the development and propagation of AML through Tetraspanin 3 (refs 24). Future studies will determine how MSI2 alter stem cells in MDS or whether it uses similar mechanisms as in AML.

Several studies have demonstrated that Msi2 is required for HSPC engraftment ${ }^{4,10}$. It is unclear if in the context of suppressed hematopoiesis where few normal HSCs remain, targeting could result in additional toxicity. However, we found that the HSPC 
$\mathbf{h}$

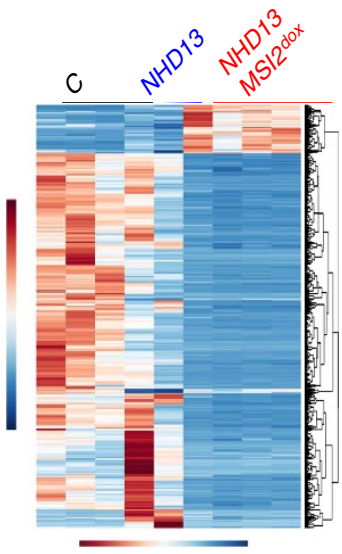

i

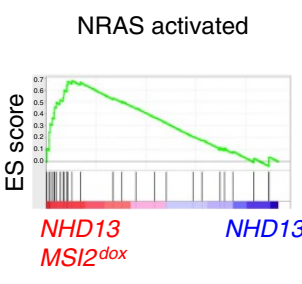

$\mathrm{FDR}=0.09 \mathrm{ES}=-0.63$ j

HSC quiescent versus dividing up

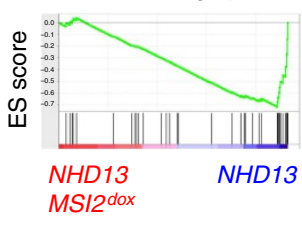

$\mathrm{FDR}=.029 \mathrm{ES}=-0.72$ $\mathbf{k}$

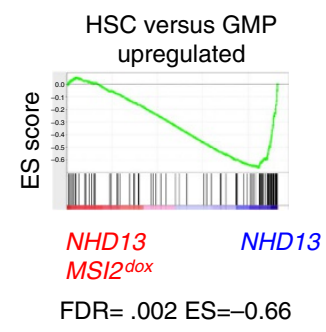

I

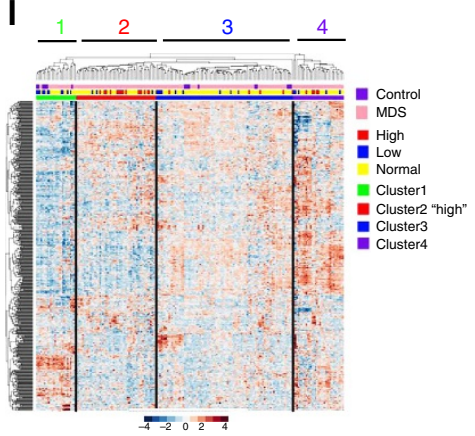

m

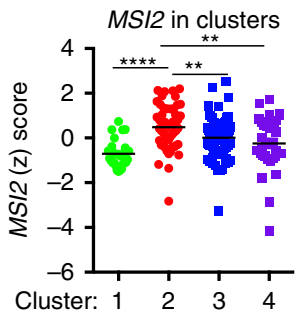

n

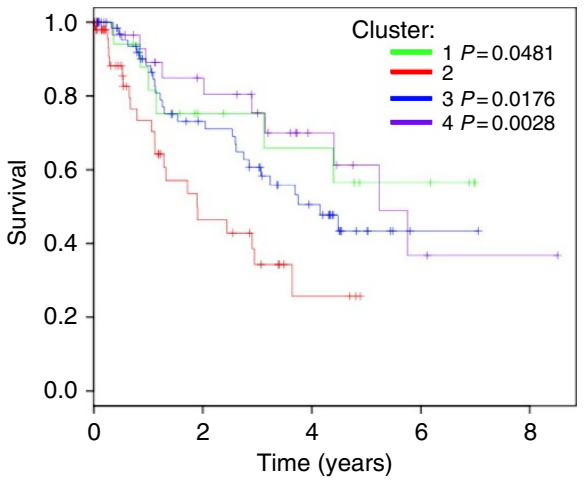

Figure 4 | continued.

population demonstrated a selective advantage and remained addicted to the forced MSI2 expression, as removal of MSI2 overexpression greatly reduced chimerism and reversed the myeloid disease. We propose that the increased expression of MSI2 in the HSPCs in high-risk MDS patients might allow for a therapeutic index in these patients. Our mouse model might provide a context to test how targeting MSI2 might alter the disease. Overall, our study suggests that targeting MSI2 could provide a therapeutic benefit in MDS.

\section{Methods}

Transgenic mice. KH2-Col1A1-tet-on-MSI2/ROSA26-rTTA transgenic mice ${ }^{5}$, backcrossed 10 times to $\mathrm{C} 57 \mathrm{BL} / 6$ strain or Msi2 conditional knockout ${ }^{4}$ were crossed with Vav-Tg-NUP98-HOXD13 mice. The primary donors that were used for transplants were either male or female of 3-4-month-old animals ${ }^{14}$. All animal procedures were approved by the Institutional Animal Care and Use Committee at Memorial Sloan Kettering Cancer Center.

Non-competitive transplants. Transplants were performed with $2-3 \times 10^{6}$ bone marrow cells from 12-16-week-old C57BL/6 donor mice mixed with $0.2 \times 10^{6}$ $\mathrm{CD} 45.1^{+}$helper cells injected into the retro-orbital of lethally irradiated B6.SJL-Ptprc ${ }^{\mathrm{a}} \mathrm{Pepc}^{\mathrm{b}} /$ BoyJ recipient mice. Secondary transplants were performed by injecting $1 \times 10^{6}$ bone marrow or spleen cells into sublethally irradiated B6.SJL-Ptprc ${ }^{\mathrm{a}} \mathrm{Pepc}^{\mathrm{b}} /$ BoyJ mice.

Peripheral blood analysis. Peripheral blood was collected from the facial vein using a lancet or retro-orbital cavity using a heparinized glass capillary tube. A compete peripheral blood count was collected using a Hemavet 950 (Drew Scientific).

Flow cytometry. Flow cytometry experiments were carried out using BD Fortessa, LSRII, or LSRFortessa instruments. Bone marrow and spleen cells collected from mice were subjected to red blood cell lysis before staining. Peripheral blood and leukaemic bone marrow and spleen were immunophenotyped with the following antibodies: CD45.2, CD45.1, Mac1, Gr1, c-Kit, CD71, Ter119 and B220. For stem cell analyses, bone marrow cells were stained with the following antibodies: lineage (Gr1, B220, CD3a, CD4, CD8 and Ter119), Sca1, c-Kit, CD150, CD48, CD16/32 and CD34. MSI2 staining was performed using a rabbit anti-mouse/human MSI2 antibody (Abcam) with a goat anti-rabbit Alexa647 conjugated secondary (Life Technologies). Anti-mouse antibodies were used at 1:200 and secondary antibodies were used at 1:400. Data analyses were performed using the FlowJo software.

Statistical analyses. To compute $P$ values for bar graphs, an unpaired 2-tailed Student's $t$-test was used except where stated otherwise. Error bars reflect the s.e.m., except where stated otherwise. In survival curves, significance was calculated using log-rank analysis. Graph Pad Prism 4.0 and the R statistical environment were used to carry out all statistical analyses.

NHD13 RNA-seq analysis. NHD13 mouse RNA-seq raw data were deposited to Gene Expression Omnibus (GEO) GSE76840. Differential analysis of RNA-seq samples utilized the DESeq package for gene expression analysis ${ }^{25}$. False discovery rate correction of $P$ values used for all bioinformatics analyses of this study utilized the Benjamini-Hochberg procedure. Mapping between entrez IDs between mouse and human genes was done using the biomaRt $\mathrm{R}$ packages ${ }^{26}$. Heatmap clustering and production was done using the heatmap function found within the NMF package in $\mathrm{R}$ (ref. 27).

Human data analysis and RNA-seq analysis. The clinical microarray samples consist of 183 and 17 healthy controls from anonymized donors that were not age matched, but included elderly patients who underwent hip replacement. MDS patients and 17 controls samples were publically available on GEO with the reference series tag: GSE19429 (ref. 12). The MDS patient samples were collected from several centres: Oxford and Bournemouth (UK), Duisburg (Germany), Stockholm (Sweden) and Pavia (Italy). This study was approved by the ethics committees (Oxford C00.196, Bournemouth 9991/03/E, Duisburg 2283/03, Stockholm 410/03, Pavia 26264/2002) and informed consent was obtained ${ }^{12}$

The microarray data were downloaded and had gene identifiers in the form of AffyID probes. For MSI2 we mapped the probes and found that only 4 out of the 9 probes were correctly matched to MSI2. We utilized the Spearman correlation coefficient to assess the correlation with the remaining probes and the three probes, which showed a good correlation (1552364_s_at, 243010_at and 243579_at), were then averaged together. These AffyID probes were converted to human and mouse 
Entrez IDs, which was done using the biomaRt tool in R. The AffyIDs that had human and mouse Entrez IDs were kept as it indicated that the AffyID corresponded to a human gene that had an orthologue in mouse. In the case that several AffyIDs mapped to a single human Entrez ID the corresponding AffyID rows were combined and a mean of their values were taken. This gave one row of mean expression of all Affy probes that corresponded to a human Entrez gene.

Kaplan-Meier curves were generated to gauge survival probability between the samples that had high, low and normal MSI2 expression. A heatmap was generated using the statistically significant genes derived from the general linearized model. The rows in this expression matrix were $\log 10$ transformed and then a $Z$-score was computed for every element in the row. A heatmap was generated using this matrix of $Z$-scores. This heatmap allowed for row and column clustering. The 200 samples which composed this matrix were labelled according to whether the sample was derived from an MDS patient or a control. Additionally, the samples were labelled according to their French-American-British MDS clinical classification. The samples could take 1 of 4 possible classifications: healthy, RA, RA with excess blasts (RAEB) and RARS. Lastly, the samples were also classified according to their MSI2 expression levels, which could be classed as high MSI2 expression, low MSI2 expression or normal MSI2 expression. MSI2 expression was classified as high if the Z-score for the MSI2 in a sample was $>1$. MSI2 expression was classified as low if the $Z$-score for the musashi2 gene in a sample was $<-1$. MSI2 expression was classified as normal if the $Z$-score for the musashi2 gene in a sample was $-1 \leq \times \leq 1$.

Age-matched normal individuals and primary MDS patient samples. Normal bone marrows from elderly individuals were obtained from hip replacements and MDS patient samples (PBMCs, low risk; RA $n=3$, RARS $n=2$ and high risk; RAEB-1 $n=4$, RAEB-2 $n=6$ ) were obtained from the Memorial Hospital Tumor Bank under the protocol IRB Waiver Number: WA0260-12 and HBUC: HBS2012060.

Cell Cycle analysis. Before $24 \mathrm{~h}$ analysis, mice received an intraperitoneal injection of $1 \mathrm{mg} \mathrm{kg}{ }^{-1}$ of BRDU. Mice were killed and Lin-Scal $+\mathrm{c}-\mathrm{Kit}+$ cells were sorted, fixed with $1.6 \%$ paraformaldehyde for $15 \mathrm{~min}$, and permeablized with ice-cold methanol. To prevent cell loss, LSKs were mixed with B220 + splenocytes and subsequently stained with CD34 and Hoechst for cell cycle, and then analysed by flow cytometry.

\section{References}

1. Shih, A. H. \& Levine, R. L. Molecular biology of myelodysplastic syndromes. Semin. Oncol. 38, 613-620 (2011).

2. Will, B. et al. Stem and progenitor cells in myelodysplastic syndromes show aberrant stage-specific expansion and harbor genetic and epigenetic alterations. Blood 120, 2076-2086 (2012).

3. Nimer, S. D. Myelodysplastic syndromes. Blood 111, 4841-4851 (2008).

4. Park, S.-M. et al. Musashi- 2 controls cell fate, lineage bias, and TGF- $\beta$ signaling in HSCs. J. Exp. Med. 211, 71-87 (2014).

5. Kharas, M. G. et al. Musashi-2 regulates normal hematopoiesis and promotes aggressive myeloid leukemia. Nat. Med. 16, 903-908 (2010).

6. Ito, T. et al. Regulation of myeloid leukaemia by the cell-fate determinant Musashi. Nature 466, 765-768 (2010).

7. Pereira, J. K. et al. Distinct expression profiles of MSI2 and NUMB genes in myelodysplastic syndromes and acute myeloid leukemia patients. Leuk. Res. 36, 1300-1303 (2012)

8. Byers, R. J., Currie, T., Tholouli, E., Rodig, S. J. \& Kutok, J. L. MSI2 protein expression predicts unfavorable outcome in acute myeloid leukemia. Blood 118, 2857-2867 (2011).

9. Park, S. M. et al. Musashi2 sustains the mixed-lineage leukemia-driven stem cell regulatory program. J. Clin. Invest. 125, 1286-1298 (2015).

10. de Andrés-Aguayo, L. et al. Musashi 2 is a regulator of the HSC compartment identified by a retroviral insertion screen and knockout mice. Blood 118, 554-564 (2011).

11. Thol, F. et al. Prognostic significance of expression levels of stem cell regulators MSI2 and NUMB in acute myeloid leukemia. Ann. Hematol. 92, 315-323 (2013).

12. Pellagatti, A. et al. Deregulated gene expression pathways in myelodysplastic syndrome hematopoietic stem cells. Leukemia 24, 756-764 (2010).

13. Raza-Egilmez, S. Z. et al. NUP98-HOXD13 gene fusion in therapy-related acute myelogenous leukemia. Cancer Res. 58, 4269-4273 (1998).

14. Lin, Y.-W., Slape, C., Zhang, Z. \& Aplan, P. D. NUP98-HOXD13 transgenic mice develop a highly penetrant, severe myelodysplastic syndrome that progresses to acute leukemia. Blood 106, 287-295 (2005).

15. Chung, Y. J., Choi, C. W., Slape, C., Fry, T. \& Aplan, P. D. Transplantation of a myelodysplastic syndrome by a long-term repopulating hematopoietic cell. Proc. Natl Acad. Sci. USA 105, 14088-14093 (2008).

16. Xu, H. et al. Loss of $\mathrm{p} 53$ accelerates the complications of myelodysplastic syndrome in a NUP98-HOXD13-driven mouse model. Blood 120, 3089-3097 (2012).
17. Subramanian, A. et al. Gene set enrichment analysis: a knowledge-based approach for interpreting genome-wide expression profiles. Proc. Natl Acad. Sci. USA 102, 15545-15550 (2005).

18. Croonquist, P. A., Linden, M. A., Zhao, F. \& Van Ness, B. G. Gene profiling of a myeloma cell line reveals similarities and unique signatures among IL-6 response, $\mathrm{N}$-ras-activating mutations, and coculture with bone marrow stromal cells. Blood 102, 2581-2592 (2003).

19. Graham, S. M., Vass, J. K., Holyoake, T. L. \& Graham, G. J. Transcriptional analysis of quiescent and proliferating CD34 + human hemopoietic cells from normal and chronic myeloid leukemia sources. Stem Cells 25, 3111-3120 (2007).

20. Gerstung, M. et al. Combining gene mutation with gene expression data improves outcome prediction in myelodysplastic syndromes. Nat. Commun. 6, 5901 (2015).

21. Humeniuk, R., Koller, R., Bies, J., Aplan, P. \& Wolff, L. Brief report: loss of p15Ink4b accelerates development of myeloid neoplasms in Nup98-HoxD13 transgenic mice. Stem Cells 32, 1361-1366 (2014).

22. Greenblatt, S. et al. Knock-in of a FLT3/ITD mutation cooperates with a NUP98-HOXD13 fusion to generate acute myeloid leukemia in a mouse model. Blood 119, 2883-2894 (2012).

23. Slape, C., Liu, L. Y., Beachy, S. \& Aplan, P. D. Leukemic transformation in mice expressing a NUP98-HOXD13 transgene is accompanied by spontaneous mutations in Nras, Kras, and Cbl. Blood 112, 2017-2019 (2008).

24. Kwon, H. Y. et al. Tetraspanin 3 is required for the development and propagation of acute myelogenous leukemia. Cell Stem Cell 17, 152-164 (2015).

25. Anders, S. \& Huber, W. Differential expression analysis for sequence count data. Genome Biol. 11, R106 (2010).

26. Durinck, S., Spellman, P. T., Birney, E. \& Huber, W. Mapping identifiers for the integration of genomic datasets with the R/Bioconductor package biomaRt. Nat. Protoc. 4, 1184-1191 (2009).

27. Gaujoux, R. \& Seoighe, C. A flexible R package for nonnegative matrix factorization. BMC Bioinformatics 11, 367 (2010).

\section{Acknowledgements}

We would like to thank Ross Levine for helpful discussions. We would like to thank Aaron Chang for experimental support. We would also like to thank Aly Azeem Khan, Agnes Viale and the MSKCC sequencing core for all their support. M.G.K. is supported by the US National Institutes of Health, National Institute of Diabetes and Digestive and Kidney Diseases; R01DK101989-01A1, National Cancer Institute R01, 1R01CA193842-01, MSK Cancer Center Support Grant/Core Grant (P30 CA008748), Louis V Gerstner Young Investigator Award, Kimmel Scholar Award and V-Scholar Award, Leukemia Research Foundation, C.J.L. was supported by an R01 from the National Cancer Institute (NIH), and a fellowship from the W.W. Smith Charitable Trust.

\section{Author contributions}

J.T., T.C.H., E.A., H.X., T.S.B., P.T., R.O., A.C., L.V., S.M.P. and C.P. performed experiments. A.R.P. and C.L. performed computational work and biostatistics. B.H.D. performed histopathology. C.F., M.P., G.R., M.G. and V.M.K. provided clinical MDS samples. C.L. provided critical reagents and discussion. A.V. provided critical project advice. S.D.N. provided support and critical advice; M.G.K. wrote the manuscript, managed the project and supported the work.

\section{Additional information}

Accession codes: NHD13 mouse RNA-seq raw data was deposited to GEO under the accession code GSE76840.

Supplementary Information accompanies this paper at http://www.nature.com/ naturecommunications

Competing financial interests: The authors declare no competing financial interests.

Reprints and permission information is available online at http://npg.nature.com/ reprintsandpermissions/

How to cite this article: Taggart, J. et al. MSI2 is required for maintaining activated myelodysplastic syndrome stem cells. Nat. Commun. 7:10739 doi: 10.1038/ncomms10739 (2016).

This work is licensed under a Creative Commons Attribution 4.0 International License. The images or other third party material in this article are included in the article's Creative Commons license, unless indicated otherwise in the credit line; if the material is not included under the Creative Commons license, users will need to obtain permission from the license holder to reproduce the material. To view a copy of this license, visit http://creativecommons.org/licenses/by/4.0/ 